La mejora de la calidad, la seguridad y el medio ambiente en la construcción de cubiertas con elementos de colocación discontinua de hormigón mediante el desarrollo y aplicación de la normativa

\section{The improvement of quality, security and environment on the installation of covers using discontinuous concrete pieces by means of the development and application of standards and regulations}

F. Marín

\section{RESUMEN}

El desarrollo de la normativa y las reglamentaciones, tanto españolas como europeas, durante las dos últimas décadas han permitido avanzar en el aseguramiento de la calidad de los materiales de hormigón para cubiertas, garantizar su seguridad, contribuyendo a la prevención de riesgos laborales en el sector de la construcción y reducir el impacto ambiental. En este trabajo se presentan los últimos avances en este campo, así como las nuevas normas de tejas de hormigón, publicadas en junio de 2005 , las cuales permiten el Marcado CE a partir de septiembre de 2005, que será obligatorio en septiembre de 2006.

\section{$585-1$}

Palabras clave: calidad, cubiertas, elementos de hormigón, marcado CE, medio ambiente, normativa en construcción, seguridad en construcción.

\section{SUMMARY}

The development of Spanish and European standards and regulations, in the last two decades, have allowed to move forward on the quality assurance of cocrete materials for roofs and covers, to guarantee their security, making a contribution to prevent labour risks in the building sector and to reduce the enviromental impact, This paper show she last advances ib this matter, and the new concrete roofing tile standars, published on june 2005, that permit the CE Marking since september 2005, that will be mandatory on september 2006.

Keywords: CE marking, concrete pieces, covers, environment, quality, security on building, standards for building.

\footnotetext{
${ }^{(*)}$ Doctor Ingeniero Químico 


\section{INTRODUCCIÓN: REGLAMENTACIÓN Y NORMATIVA}

La legislación regula con carácter obligatorio determinadas actividades, así como las características de algunos productos, especialmente en lo que afecta a garantizar la seguridad y la salud de los ciudadanos. La legislación tiene dos utilidades:

Concreta el marco de deberes y derechos.

Da orientaciones técnicas concretas a aplicar en algunos casos.

El poder legislativo reside en el Parlamento, que elabora y aprueba las leyes. Del Gobierno emanan los Decretos y órdenes ministeriales, que deben respetar la ley de la que derivan o a la que complementan y los Decretos-leyes, que con carácter de ley ordinaria (nunca orgánica), pueden emitirse por causa de extraordinaria y urgente necesidad y deben ser convalidadas por el Parlamento en el plazo máximo de 30 días.

Los tratados internacionales forman parte del derecho nacional una vez suscritos y ratificados.

La Unión Europea está configurando, mediante Directivas, un marco donde las condiciones de trabajo de los distintos países son cada vez más similares y los productos comercializados cumplen idénticos requisitos de seguridad. Las Directivas comunitarias son adaptadas por cada país europeo a su legislación mediante «transposición».

Una Norma es un documento de aplicación voluntaria que contiene especificaciones técnicas basadas en los resultados de la experiencia y del desarrollo tecnológico; es el fruto del consenso entre todas las partes interesadas e involucradas en la actividad objeto de la misma, debe aprobarse por un Organismo de Normalización reconocido.

Las normas son voluntarias, únicamente son de obligado cumplimiento cuando:

Se haga referencia a ellas en Leyes, Reglamentos, Códigos Técnicos, Instrucciones Técnicas Complementarias $\mathrm{u}$ otros documentos de carácter reglamentario.

Sean "armonizadas", en desarrollo de Directivas Europeas. En ese caso sólo es obligatoria la parte armonizada, el resto es voluntario.

Haya acuerdo contractual sobre el cumplimiento de ciertas normas, por ejemplo en acuerdos comerciales.

Los organismos de normalización, que elaboran y aprueban normas pueden ser:
Nacionales: AENOR en España, AFNOR en Francia, BS en Reino Unido, DIN en Alemania, UNI en Italia, etc.

Europeos: CEN, sus normas son obligatoriamente adoptadas por los 25 países de la Unión Europea y otros signatarios, caso de Suiza, para evitar las barreras técnicas al libre comercio en el Mercado Común. Algunas normas europeas son «mandatadas» por la Comisión Europea a CEN en desarrollo de una Directiva, en este caso, se dice que la norma europea es armonizada.

Internacionales: ISO, sus normas pueden ser adoptadas por cualquier país.

\section{DESARROLLO DE LA NORMATIVA Y REGLAMENTACIÓN PARA TEJADOS CON ELEMENTOS DE HORMIGÓN}

\subsection{Prefabricados de hormigón para tejados}

Los prefabricados de hormigón se utilizan en los tejados como elementos estructurales (placas y forjados), como elementos soporte (tableros), de cobertura discontinua (tejas y piezas) y en otras aplicaciones especiales (conductos de humos, chimeneas, etc.).

Las tejas de hormigón son susceptibles de utilización en obra nueva, rehabilitación y entornos históricos protegidos, en pendientes que van desde el $26 \%$ (15\%) hasta la vertical (véanseFiguras 1 y 2), aportando las siguientes características (Marín, 2004):

Excelentes propiedades mecánicas, de resistencia a la helada, impermeabilidad, estabilidad dimensional y resistencia al fuego.

Efecto estético adaptado a los requerimientos: colores homogéneos, envejecidos, texturas lisas y rugosas, vidriados, aspecto de crecimiento de musgo y líquenes, etc. Capaces de sustituir e imitar a las tejas cerámicas curvas y a las pizarras,.

Facilidad de montaje y posibilidad de formar parte de un sistema de tejados como unidad de obra con elementos industrializados (Cobos et al. 2005).

Mayor superficie de cubrición que las tejas de otros materiales, y en particular, las curvas.

La gama de piezas especiales, complementarias de las tejas, solucionan los puntos singulares del tejado y resuelven exigencias de seguridad, a la 
The improvement of quality, security and environment on the installation of covers using discontinuous concrete pieces by means of the development and application of standards and regulations

vez que mejoran las prestaciones y ornamento del tejado, permitiendo el montaje en seco.

Por eso, el mercado de tejas y piezas de hormigón ha ido desarrollándose en la Unión Europea, a la vez que se han ido introduciendo innovaciones para mejorar sus características y desarrollar nuevos acabados y modelos.

\subsection{Desarrollo de la reglamentación}

La primera reglamentación española elaborada para cubiertas con elementos de colocación discontinua fue la Norma Tecnológica de la Edificación NTE-QTT Tejados de Tejas, publicada en los Boletines Oficiales del Estado (B.O.E.) de fecha 14-12-1974 y 21-12-1974.

Actualmente, está pendiente de ser publicado el Código Técnico de la Edificación, CTE, en desarrollo de la Ley de Ordenación de la Edificación, LOE, que actualizará las obsoletas Normas Tecnológicas de la Edificación.

En 1989 fue aprobada la Directiva Comunitaria 89/106/CEE Productos de Construcción, DPC, cuyos requisitos esenciales, que deben cumplir los materiales de construcción, son:

1. Resistencia mecánica y estabilidad.

2. Seguridad en caso de incendio.

3. Higiene, salud y medio ambiente.

4. Seguridad de utilización.

5. Protección contra el ruido.

6. Ahorro de energía y aislamiento térmico.

Existen dos procedimientos para asegurar que los distintos productos de la construcción cumplen con los requisitos esenciales de las directivas:

a) Para productos tradicionales, la elaboración de normas CEN armonizadas, v.gr. UNE-EN 490 para tejas de hormigón, que incluyen un anexo ZA que especifica cuáles de los requisitos de la norma son de obligado cumplimiento para satisfacer la directiva.

b) Para productos innovadores o sistemas, los Documentos de Idoneidad Técnica Europea, DITE, que elaboran los organismos de la EOTA, cuyo representante español es el Instituto Eduardo Torroja del CSIC.

Los productos de construcción que cumplan las normas armonizadas o dispongan de un DITE, ostentarán el Marcado CE y podrán comercializarse en la Unión Europea.

\subsection{Desarrollo de la normativa}

La primera norma aplicable a las tejas de hormigón en España fue la UNE 41200 “Tejas de

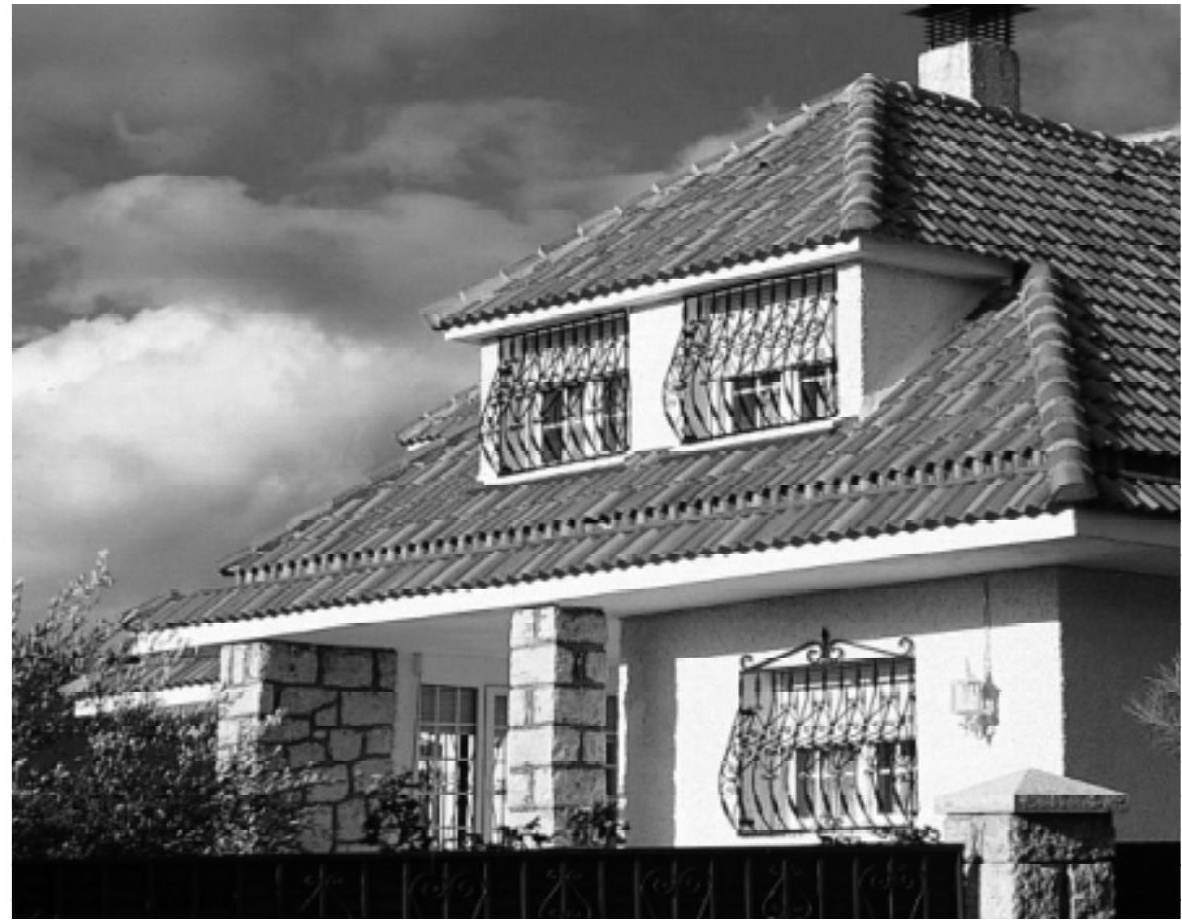

Figura 1. Vivienda con tejas de hormigón de aspecto rojo envejecido.

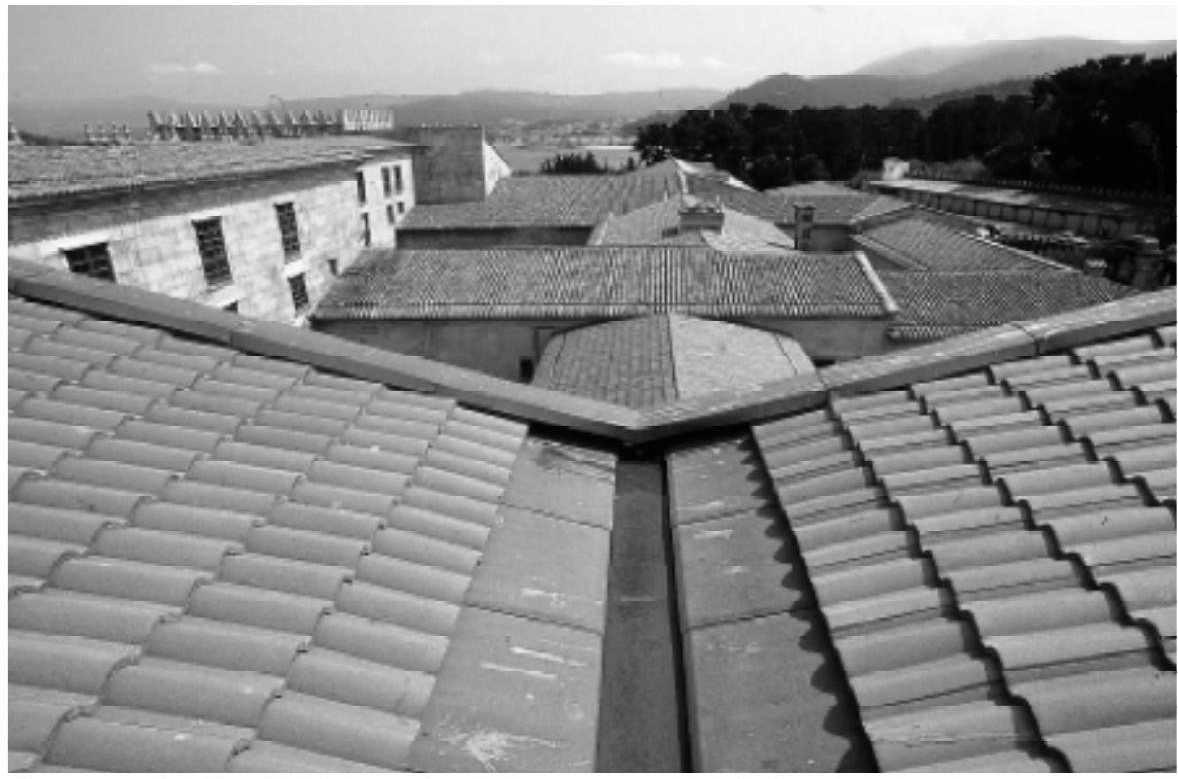

Figura 2. Rehabilitación del parador de Bayona con tejas de hormigón.

hormigón. Clasificación. Características y métodos de ensayo" publicada en junio de 1988, la cual se basaba en experiencia propia y en otras normas sobre el mismo sujeto de países extranjeros, siendo ya en aquel momento patente las elevadas exigencias normativas a los productos de hormigón con respecto a otros materiales de uso en cubiertas (Tascón et al. 1994).

La norma UNE 41200 tuvo una primera enmienda en julio de 1994, para facilitar el tránsito a la nueva normativa europea que la sustituiría en febrero de 1995, consistente en dos documentos: 
Norma UNE-EN 490 "Tejas y accesorios de hormigón. Especificaciones de producto"

Norma UNE-EN 491 "Tejas y accesorios de hormigón. Métodos de ensayo"

Por mandato de la Comisión Europea, el Comité TC128 de CEN revisó las normas anteriores, para armonizarlas a la DPC, a la vez que las completó para incluir especificaciones y ensayos de piezas, siendo publicadas las nuevas normas en diciembre de 2004, habiendo sido adoptadas por AENOR y publicadas en español en junio de 2005, con los títulos:

UNE-EN 490 «Tejas y piezas de hormigón para tejados y revestimiento de muros. Especificaciones de producto»

UNE-EN 491 «Tejas y piezas de hormigón para tejados y revestimiento de muros. Métodos de ensayo.»

Esta normativa se completará con la norma en desarrollo para otros prefabricados de hormigón para tejados EN 13693 "Special precast prestressed concrete roof elements".

En cuanto al diseño de las cubiertas y al montaje de las tejas, la primera norma española, basada en la experiencia propia y en otras normativas europeas, fue elaborada por el comité técnico del AEN CTN 127, se publicó en febrero de 1999 con el título UNE 127100 "Tejas de hormigón. Código de práctica para la concepción y el montaje de cubiertas con tejas de hormigón", la cual especifica cómo deben de colocarse las tejas en cubierta para asegurar la funcionalidad y calidad del conjunto, a la vez que da instrucciones para garantizar la seguridad de los instaladores, estando vigente en la actualidad y habiendo sido referencia, por su novedad, para la elaboración de códigos de práctica similares de otros materiales para tejados (Marín, 1999).

\section{APORTACIONES DE LA NORMATIVA PARA TEJAS DE HORMIGÓN DE 2005}

La nueva normativa para tejas de hormigón, compuesta por las normas UNE-EN 490 y UNEEN 491 de junio de 2005, supone los siguientes cambios:

revisión y actualización de la normativa europea anterior, inclusión de requisitos y métodos de ensayo para las piezas complementarias a las tejas, que habían ido ganando importancia y mercado al solucionar los puntos singulares del tejado, resolver exigencias de seguridad, a la vez que proporcionan nuevas prestaciones y ornamento al tejado y

armonización a la Directiva de Productos de Construcción teniendo en consideración aspectos reglamentarios de seguridad, al obligar al cumplimiento de los requisitos esenciales de la Directiva, el establecimiento de un sistema de Control de Producción en Fábrica para asegurar la calidad y propiedades del producto, así como la toma en consideración de las reglamentaciones nacionales sobre liberación de sustancias peligrosas y de protección del medio ambiente.

En la Tabla 1 se muestra la comparación entre la nueva normativa de tejas y piezas de hormigón de 2005 y la precedente de 1995 . En la Tabla 2 se detallan las especificaciones de resistencia a flexión transversal en función del tipo de tejas y de sus dimensiones.

Puede observarse que las principales novedades de la normativa de 2005 se refieren a la inclusión del comportamiento frente al fuego, que se considera desde los puntos de vista de comportamiento frente al fuego exterior y reacción al fuego, aceptándose en ambos casos que el material, hormigón, por su naturaleza y cualidades cumple los requisitos, excepto en el caso de tener materia orgánica o incluir otro tipo de materiales, así como a considerar la liberación de sustancias peligrosas, que a medida que se desarrolle la normativa al respecto irá incluyendo restricciones al uso de materias que puedan ser dañinas para la salud o el medio ambiente.

Otra importante novedad es que se establecen requisitos a las piezas complementarias, a las que anteriormente sólo se les pedía que tuvieran la misma composición material que las tejas y se establecía el requisito de impermeabilidad de las piezas de limahoya.

Por otra parte, la norma UNE-EN 490 añade un anexo ZA en el que se establecen las especificaciones mínimas que deben cumplirse, de entre las establecidas en la norma, para satisfacer los requisitos esenciales de la DPC en los diferentes usos previstos de los productos.

Se han realizado algunas ligeras modificaciones en los métodos de ensayo, donde lo más relevante es el cambio del concepto de resistencia característica, que conllevaba la asunción de cálculos estadísticamente discutibles, por el de resistencia mínima y la posibilidad de que, para el control de producción en fábrica y, si fuera pertinente, para el control por tercera parte, ensayo de un suministro o inspección, el ensayo puede llevarse a cabo sin 
La mejora de la calidad, la seguridad y el medio ambiente en la construcción de cubiertas con elementos de colocación discontinua de hormigón mediante el desarrollo y aplicación de la normativa

The improvement of quality, security and environment on the installation of covers using discontinuous concrete pieces by means of the development and application of standards and regulations

utilizar la almohadilla de elastómero o la pieza contorneada (véase Figura 3), lo cual aproxima la metodología del ensayo a las condiciones de carga que soportan las tejas en las cubiertas cuando se transita sobre ellas.

También se ha modificado y completado el sistema de designación de las tejas.

\section{CERTIFICACIÓN DE PRODUCTO: MARCADO CE Y MARCA N DE CALIDAD}

Las tejas y piezas de hormigón se están certificando en España con Marca AENOR, que garantiza la Calidad del producto y el cumplimiento de la normativa vigente, desde el año 1995, fecha en que fueron pioneras las fábricas del Grupo Uralita. En la actualidad cerca del $95 \%$ de las tejas y piezas de hormigón fabricadas en España tienen esta certificación de calidad, lo que garantiza al montador y al usuario final la calidad del producto, su adecuación a la obra de construcción y una mayor seguridad de manejo (Cobos et al. 2000).

El Marcado CE no es una marca de calidad voluntaria, supone el reconocimiento, que, con carácter obligatorio, debe realizar el fabricante o comercializador, del cumplimiento de los mínimos de seguridad establecidos por los requisitos esenciales de la Directiva de Productos de la Construcción, recogidos en el anexo ZA de las normas armonizadas. En el caso de las tejas y piezas de hormigón el Marcado CE comenzó con carácter voluntario el día 1 de septiembre de 2005 y será obligatorio para poder vender los productos en España y en cualquier otro Estado de la Unión Europea a partir del día 1 de septiembre de 2006. A partir de esa fecha, si un fabricante no dispone de Marcado CE podría seguir fabricando sus productos, pero no los podría vender en el seno de la Unión Europea, aunque no tendría problemas para venderlos en mercados terceros tales como Argelia, Marruecos, etc.

En la Tabla 3 se recoge una comparación entre los requisitos y los controles establecidos por la certificación de Marca de Calidad y por el Marcado CE para tejas y piezas de hormigón. En esta Tabla puede comprobarse que las principales diferencias estriban en:

La Marca de Calidad exige al fabricante una mayor frecuencia de ensayos y un número mayor de muestras, así numerosas características se ensayan una vez cada 7 días a efectos de Marcado CE, mientras que la Marca AENOR exige en ensayo diario de cada modelo fabricado, lo que supone, en una fábrica tipo con 3 modelos que trabaje 5 días a la semana, realizar 3 ensayos diarios y un total de 15 semanales.

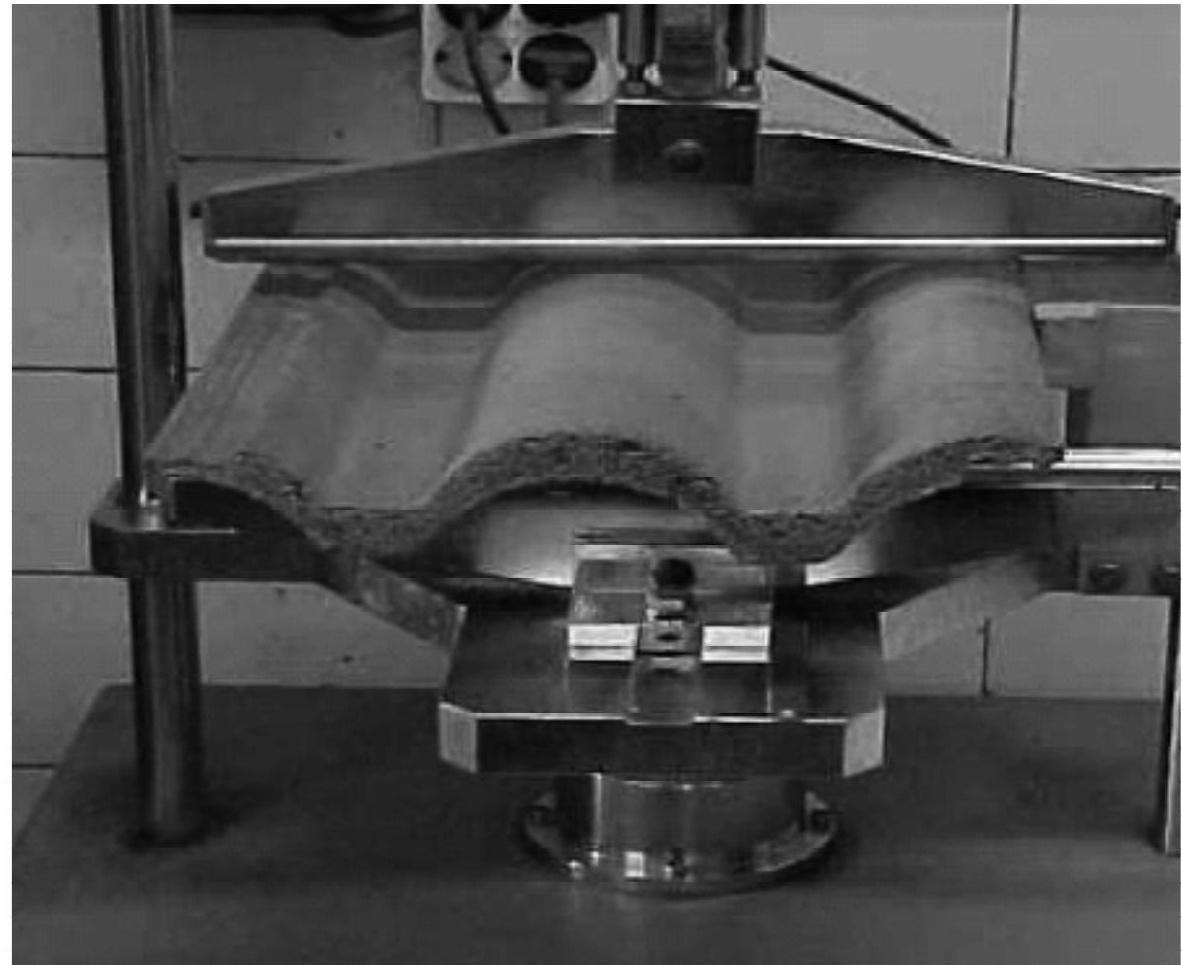

Figura 3. Ensayo de resistencia a flexión transversal.

Características como la resistencia al hielo-deshielo y el soporte por el tacón se consideran de tipo a efectos de Marcado CE, mientras que la Marca AENOR exige, además del ensayo de tipo, su ensayo anual.

El Marcado CE exige al fabricante disponer de un sistema de Control de la Producción en Fábrica, CPF, pero no especifica nada más; la Marca AENOR exige disponer de un sistema de gestión de calidad basado en las especificaciones que tienen que ver con la conformidad de producto y satisfacción de clientes de la norma UNE-EN-ISO-9001:2000, que además son objeto de auditoría periódica.

El Marcado CE únicamente exige una declaración del fabricante, sin auditoria por tercera parte ni ensayos en laboratorios acreditados, excepto en el caso de no cumplir con las limitaciones para fuego por añadir material orgánico al hormigón, mientras que la Marca AENOR exige auditorías o inspecciones anuales en las que se toman muestras de los productos a efectos de ser ensayados en laboratorios acreditados.

Tanto la Marca AENOR como el Marcado CE tienen previsto la vigilancia de mercado, sin embargo, ni AENOR ni la administración realizan sistemáticamente dicha vigilancia, actuando únicamente en caso de reclamaciones o denuncias. 
Tabla 1

Comparación entre la normativa de 2005 y la de 1995

\begin{tabular}{|c|c|c|c|}
\hline \multirow{2}{*}{\multicolumn{2}{|c|}{ ESPECIFICACIONES }} & \multicolumn{2}{|c|}{ REQU ISITOS } \\
\hline & & UNE-EN 490:2005 & UNE-EN 490-1995 \\
\hline \multirow{13}{*}{$\frac{2}{13}$} & Materiales & Hormigón: cemento, áridos y agua & Hormigón: cemento, áridos y agua \\
\hline & $\begin{array}{l}\text { Longitud de cuelgue y perpendicularidad en } \\
\text { tejas de frente regular }\end{array}$ & $\begin{array}{l}\text { Tolerancia } \pm 4 \text { mm en longitud de } \\
\text { cuelgue o descuadre }\end{array}$ & $\begin{array}{l}\text { Tolerancia } \pm 4 \text { mm en longitud de } \\
\text { cuelgue o descuadre }\end{array}$ \\
\hline & Anchura efectiva en tejas con ensamble & $\begin{array}{l}\text { Tolerancia } \pm 5 \mathrm{~mm} \text { sobre el valor } \\
\text { declarado, en caso de declararse } \\
\text { holguras, debe cumplirse: } \\
\text { Cwd } / 10 \geq \mathrm{Cw}+\text { holgura máxima } \\
\text { declarada } \\
\text { Cwc } / 10 \leq \mathrm{Cw} \text { - holgura minima } \\
\text { declarada }\end{array}$ & $\begin{array}{l}\text { Tolerancia } \pm 5 \mathrm{~mm} \text { sobre el valor } \\
\text { declarado, en caso de declararse } \\
\text { holguras, debe cumplirse: } \\
\text { Cwd } 10 \geq \mathrm{Cw}+\text { holgura máxima } \\
\text { declarada } \\
\text { Cwc } 10 \leq \mathrm{Cw} \text { - holgura minima } \\
\text { declarada }\end{array}$ \\
\hline & Anchura efectiva en tejas sin ensamble & $\begin{array}{l}\text { Tolerancia } \pm 3 \mathrm{~mm} \text { sobre el valor } \\
\text { declarado }\end{array}$ & $\begin{array}{l}\text { Tolerancia } \pm 3 \mathrm{~mm} \text { sobre el valor } \\
\text { declarado }\end{array}$ \\
\hline & Planeidad (si procede) & $\begin{array}{l}\text { Holgura no superior al mayor de los } \\
\text { valores } 3 \mathrm{~mm} \text { o } \mathrm{Cw} / 100 \text {. }\end{array}$ & $\begin{array}{l}\text { Holgura no superior al mayor de los } \\
\text { valores } 3 \mathrm{~mm} \text { o } \mathrm{Cw} / 100 \text {. }\end{array}$ \\
\hline & Masa & $\begin{array}{l}\text { a) En tejas con masa declarada } \leq 2 \\
\mathrm{~kg} \text {, tolerancia en masa media de } \\
\pm 0,2 \mathrm{~kg} \text {. } \\
\text { b) En tejas con masa declarada }>2 \\
\mathrm{~kg}, \text { tolerancia en masa media de } \\
\pm 10 \%\end{array}$ & $\begin{array}{l}\text { e) En tejas con masa declarada } \leq \\
2 \mathrm{~kg} \text {, tolerancia en masa media } \\
\text { de } \pm 0.2 \mathrm{~kg} \text {. } \\
\text { d) En tejas con masa declarada }> \\
2 \mathrm{~kg}, \text { tolerancia en masa media } \\
\text { de } \pm 10 \%\end{array}$ \\
\hline & Resistencia a flexión transversal & $\begin{array}{l}\text { E valor de resistencia minima } \mathrm{F}_{\text {mi }} \\
\text { superior al de la tabla } 2 \text { para cada tipo } \\
\text { de teja }\end{array}$ & $\begin{array}{l}\text { E valor de resistencia caracteristica } \\
\mathrm{F}_{c} \text { supcrior al de la tabla } 2 \text { para cada } \\
\text { tipo de teja }\end{array}$ \\
\hline & Impermeabilidad & $\begin{array}{l}\text { Sin caida de gota durante las } 20 \mathrm{~h} \text { del } \\
\text { ensayo }\end{array}$ & $\begin{array}{l}\text { Sin caida de gota durante las } 20 \mathrm{~h} \\
\text { del ensayo }\end{array}$ \\
\hline & $\begin{array}{l}\text { Durabilidad: } \\
\text { Resistencia al hielo-deshielo }\end{array}$ & $\begin{array}{l}\text { Cumplimiento de las prescripciones } \\
\text { de impermeabilidad y resistencia a } \\
\text { flexión transversal, tras el ensayo de } \\
\text { resistencia al hielo-deshielo con } 25 \\
\text { ciclos de }+20 \text { a }-20^{\circ} \mathrm{C}\end{array}$ & $\begin{array}{l}\text { Cumplimiento de las prescripciones } \\
\text { de impermeabilidad y resistencia a } \\
\text { flexión transversal, tras el ensayo de } \\
\text { resistencia al hielo-deshielo con } 25 \\
\text { ciclos de }+20 \text { a }-20^{\circ} \mathrm{C}\end{array}$ \\
\hline & Soporte por el tacón & $\begin{array}{l}\text { Mantenimiento de las tejas durante el } \\
\text { tiempo de ensayo ( } 1 \text { minuto) }\end{array}$ & $\begin{array}{l}\text { Mantenimiento de las tejas durante } \\
\text { el tiempo de ensayo ( } 1 \text { minuto) }\end{array}$ \\
\hline & Comportamiento frente al fuego exterior & $\begin{array}{l}\text { Satisfacen los requisitos, cualquier } \\
\text { recubrimiento externo debe ser } \\
\text { inorgánico o tener un poder calorifico } \\
\text { superior }(\mathrm{PCS}) \leq 4,0 \mathrm{MJ} / \mathrm{m}^{2} \text { o una masa } \\
\$ 200 \mathrm{~g} / \mathrm{m}^{2} \text {. }\end{array}$ & \\
\hline & Reacción al fuego & $\begin{array}{l}\text { Clase Al siempre que: } \\
\text { en las tejas realizadas mediante } \\
\text { pegado de uno o más trozos de } \\
\text { hormigón, el contenido orgánico del } \\
\text { adhesivo endurecido es } \leq 0,1 \% \text { en } \\
\text { peso o volumen (cualquiera que sea el } \\
\text { menor), y contienen } 51,0 \% \text { en peso } \\
\text { o volumen (cualquiera que sea el } \\
\text { menor) de materia orgánica } \\
\text { distribuida homogéneamente, aparte } \\
\text { del adhesivo. }\end{array}$ & \\
\hline & Liberación de sustancias peligrosas & Se cumplirá la legislación aplicable & \\
\hline
\end{tabular}


La mejora de la calidad, la seguridad y el medio ambiente en la construcción de cubiertas con elementos de colocación discontinua de hormigón mediante el desarrollo y aplicación de la normativa

The improvement of quality, security and environment on the installation of covers using discontinuous concrete pieces by means of the development and application of standards and regulations

\section{Continuación}

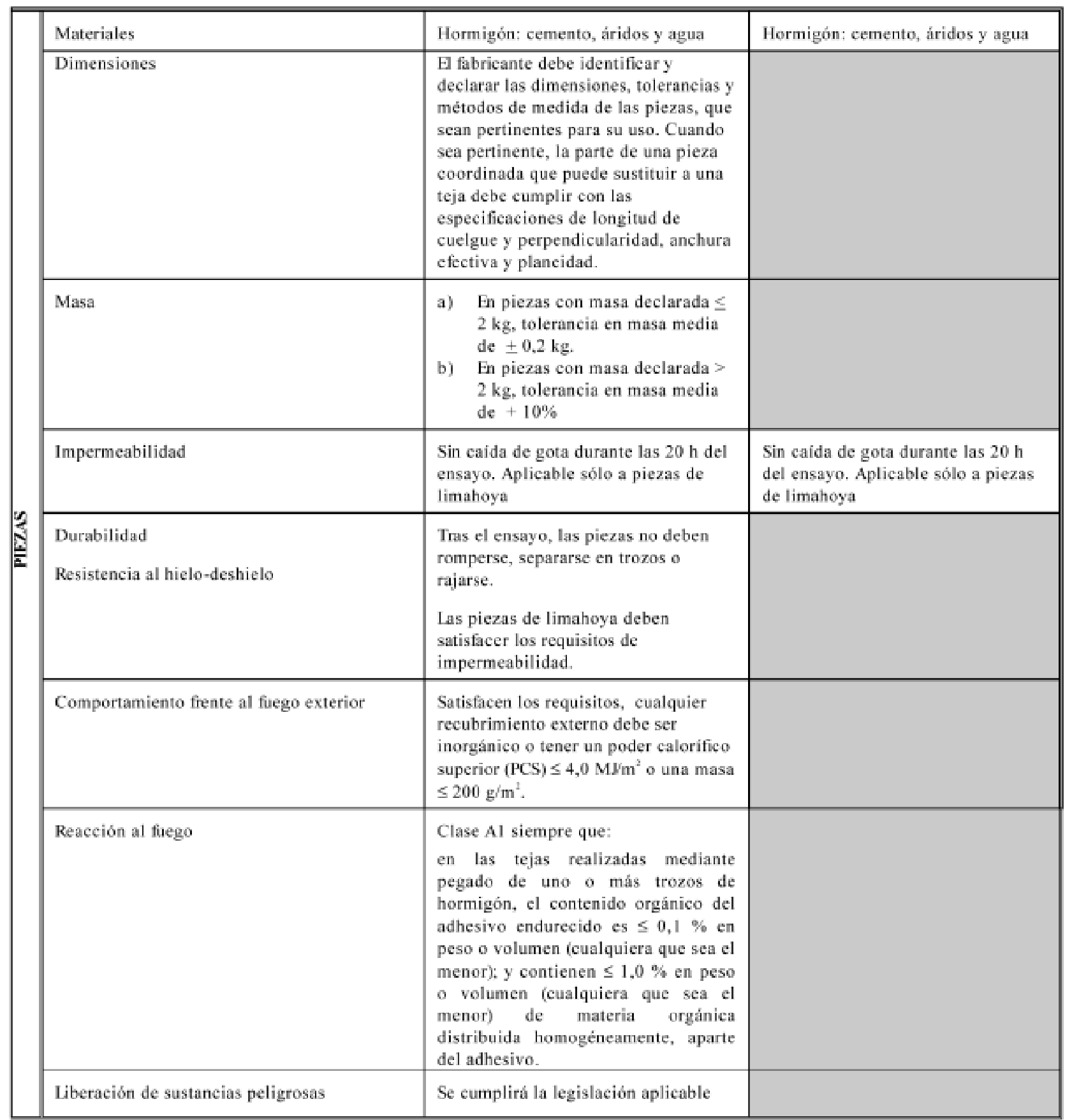

Tabla 2

Resistencia transversal de las tejas

\begin{tabular}{|c|c|c|c|c|c|c|c|}
\hline \multirow[t]{2}{*}{ Propiedad } & \multicolumn{6}{|c|}{ Tejas con ensamble } & \multirow{4}{*}{$\begin{array}{l}\text { Tejas sin } \\
\text { ensamble }\end{array}$} \\
\hline & \multicolumn{4}{|c|}{ Perfiladas } & \multicolumn{2}{|c|}{ planas } & \\
\hline Altura de onda & \multicolumn{2}{|c|}{$d>20 \mathrm{~mm}$} & \multicolumn{2}{|c|}{$20 \mathrm{~mm} \geq d \geq 5 \mathrm{~mm}$} & \multicolumn{2}{|c|}{$d<5 \mathrm{~mm}$} & \\
\hline$c_{\mathrm{w}}(\mathrm{mm})$ & $>300$ & $<200$ & $>300$ & $<200$ & $>300$ & $<200$ & \\
\hline$F_{\min }(\mathrm{N})$ & 2000 & 1400 & 1400 & 1000 & 1200 & 800 & 550 \\
\hline
\end{tabular}


Tabla 3

Comparación Marcado CE-Marca N de Calidad AENOR

\begin{tabular}{|c|c|c|c|c|c|}
\hline \multicolumn{2}{|c|}{ CERTIFICACIÓN } & \multicolumn{2}{|c|}{ Mareado CE } & \multicolumn{2}{|c|}{ Marea de Calidad AENOR } \\
\hline \multicolumn{2}{|c|}{ Carácter } & \multicolumn{2}{|c|}{ Obligatorio } & \multicolumn{2}{|c|}{ Voluntario } \\
\hline \multicolumn{2}{|c|}{$\begin{array}{l}\text { Ensayos de control de } \\
\text { producción en fábrica }\end{array}$} & $\begin{array}{c}\text { Tamaño de } \\
\text { muestra }\end{array}$ & Frecuencia minima & $\begin{array}{c}\text { Tamaño de } \\
\text { muestra }\end{array}$ & Frecuencia minima \\
\hline \multicolumn{2}{|c|}{$\begin{array}{l}\text { Longitud de cuelgue y } \\
\text { perpendicularidad }\end{array}$} & 3 & $\begin{array}{l}1 \text { cada } 7 \text { dias de } \\
\text { producción }\end{array}$ & 5 & $\begin{array}{l}\text { I por modelo y dia de } \\
\text { producción }\end{array}$ \\
\hline \multirow{2}{*}{$\begin{array}{l}\text { Dimensiones } \\
\text { de piczas }\end{array}$} & $\begin{array}{l}\text { Piezas } \\
\text { coordinadas }\end{array}$ & 3 & $\begin{array}{l}1 \text { cada } 7 \text { dias de } \\
\text { producción }\end{array}$ & 5 & $\begin{array}{l}1 \text { por modelo y dia de } \\
\text { producción (a) }\end{array}$ \\
\hline & $\begin{array}{l}\text { Piczas no } \\
\text { coordinadas }\end{array}$ & \multicolumn{2}{|c|}{ No debe ensayarse } & 5 & $\begin{array}{l}1 \text { por modelo y dia de } \\
\text { producción }\end{array}$ \\
\hline \multicolumn{2}{|c|}{ Anchura efectiva } & 11 & $\begin{array}{c}1 \text { cada } 7 \text { dias de } \\
\text { producción }\end{array}$ & 11 & $\begin{array}{l}1 \text { por modelo y dia de } \\
\text { producción }\end{array}$ \\
\hline \multicolumn{2}{|c|}{ Planeidad } & 3 & $\begin{array}{l}1 \text { cadả } 7 \text { dias de } \\
\text { producción }\end{array}$ & 5 & $\begin{array}{l}1 \text { por modelo y dia de } \\
\text { producción }\end{array}$ \\
\hline \multicolumn{2}{|c|}{ Masa } & 3 (b) & $\begin{array}{l}1 \text { cada } 7 \text { dias de } \\
\text { producción (b) }\end{array}$ & 5 & $\begin{array}{l}1 \text { por modelo y dia de } \\
\text { producción }\end{array}$ \\
\hline \multicolumn{2}{|c|}{ Resistencia a flexión transversal } & 3 & $\begin{array}{l}1 \text { cada } 7 \text { dias de } \\
\text { producción }\end{array}$ & 5 & $\begin{array}{l}\text { I por modelo y día de } \\
\text { producción }\end{array}$ \\
\hline \multicolumn{2}{|c|}{ Impermeabilidad } & 1 & $\begin{array}{c}1 \text { cada } 7 \text { dias de } \\
\text { producción }\end{array}$ & 1 & $\begin{array}{l}1 \text { por modelo y dia de } \\
\text { producción }\end{array}$ \\
\hline \multicolumn{2}{|c|}{ Resistencia al hielo-deshielo } & \multicolumn{2}{|c|}{ No debe ensayarse } & 5 & 1 por año \\
\hline \multicolumn{2}{|c|}{ Soporte por el tacón } & \multicolumn{2}{|c|}{ No debe ensayarse } & 3 & I por año \\
\hline \multicolumn{2}{|c|}{$\begin{array}{l}\text { Fisayos de comportamiento } \\
\text { frente al fuego }\end{array}$} & \multicolumn{2}{|c|}{ No debe ensayarse (c) } & \multicolumn{2}{|c|}{ No debe ensayarse } \\
\hline \multicolumn{2}{|c|}{ Sistema de calidad } & \multicolumn{2}{|c|}{$\begin{array}{l}\text { Control de Producción en Fábrica, CPF, } \\
\text { sin especificar ni auditar }\end{array}$} & \multicolumn{2}{|c|}{$\begin{array}{l}\text { Sistema de control basado en norma } \\
\text { ISO-9001:2000 inspeccionado o } \\
\text { auditado anualmente por AFNOR }\end{array}$} \\
\hline \multicolumn{2}{|c|}{ Inspección de producto } & \multicolumn{2}{|c|}{ Sin inspección de producto en fäbrica } & \multicolumn{2}{|c|}{$\begin{array}{l}\text { Inspección y ensayo de productos } \\
\text { tomados al azar en laboratorios } \\
\text { acreditados }\end{array}$} \\
\hline
\end{tabular}

(a) Se ensaya la pieza base con el mismo sistema de las tejas. En caso de no fabricarse a partir de una teja, se realiza el control sobre 3 piezas con la frecuencia establecida por el Marcado CE

(b) E ensayo de masa es opcional a efectos del Control de Producción en Fábrica para Marcado CE

(c) $\mathrm{B}$ comportamiento al fuego no es objeto de ensayos, ni de tipo ni de control de producción en fábrica, por no existir actualmente en España requerimiento legal al respecto. En caso de que, en el futuro, existiera tal requerimiento legal o de que los productos se destinaran a la venta en un Estado de la Unión Europea en que existiera tales requerimientos legales, deberian realizarse ensayos de tipo únicamente si el hormigón llevara materia orgánica en proporciones tales que el ensayo fuera preciso (véase tabla 1).

A la vista de esta comparación podría desprenderse la conclusión de que el Marcado CE no aporta nada nuevo en cuanto a la calidad de los productos. Nada más lejos de la realidad, puesto que, con respecto a la situación precedente, el Marcado CE representa unas mayores garantías en cuanto a:

Seguridad en caso de incendio. Eliminación de sustancias peligrosas, que eventualmente pudieran utilizarse. Además, la lista de sustancias peligrosas puede ir creciendo a medida que se conozca el efecto de algunas de las que actualmente no conocemos su toxicidad.

Utilización de materias primas controladas, que a su vez deberán tener Marcado CE. Este es el caso de los áridos, que tradicionalmente no tenían ningún tipo de Marca de Calidad ni control, aparte del acordado con el proveedor, y que ahora precisan el Marcado CE, de acuerdo con exigentes normas europeas. Todos los productos del mercado, que deben tener Marcado CE, pueden ser sometidos a vigilancia por las autoridades autonómicas o central, ya sea por denuncia o por vigilancia de oficio.

\section{CONCLUSIONES}

El desarrollo de la normativa y de las reglamentaciones ha sido reflejo y motor para el aseguramiento de la calidad de los materiales de hormigón para cubiertas, con el fin de garantizar su seguridad y reducir el impacto ambiental, a la vez que han permitido establecer las bases para ofrecer al mercado productos certificados con diferentes niveles de garantía, que afectan a los mínimos 
La mejora de la calidad, la seguridad y el medio ambiente en la construcción de cubiertas con elementos de colocación discontinua de hormigón mediante el desarrollo y aplicación de la normativa

The improvement of quality, security and environment on the installation of covers using discontinuous concrete pieces by means of the development and application of standards and regulations

legales de seguridad (Marcado CE) o a la calidad del producto, con un mayor rigor en el control de producción y la satisfacción del cliente (marcas de Calidad).
Su importancia queda patente en el elevado porcentaje de productos certificados existentes en el mercado español.

\section{BIBLIOGRAFÍA}

F. Cobos, F. Marín: «Problemática en tejados y fachadas de ladrillo visto». Arte y Cemento, 1889 (2000), pp. 92-96.

F. Cobos, A. Baeza, I. Iriarte, F. Marín: "Desarrollo de sistemas constructivos para tejados". Parte del libro I Jornadas de Investigación en Construcción. Actas de las Jornadas, Tomo I (2005), pp. 515-528. F. Marín: «Normas UNE de colocación de tejas de hormigón». Arte y Cemento, 1868 (1999), pp. 106108.

F. Marín: «Rehabilitación en tejados: materiales y seguridad en la ejecución». Parte del libro IV Semana de la Ciencia Madrid-2004. Seminario Restauración de cubiertas y fachadas de edificios históricos (2004), pp.2-14.

C. Tascón, F. Marín: 1994 «Las tejas de hormigón». Cemento-Hormigón, 735 (1994), pp. 897-913.

Normativa y Reglamentación

Directiva Comunitaria 89/106/CEE Productos de Construcción (DPC).

Norma Tecnológica de la Edificación NTE-QTT Tejados de Tejas, BOE 14-12-1974 y 21-121974.

UNE 41200:1988 “Tejas de hormigón. Clasificación. Características y métodos de ensayo" y $1 \stackrel{\text { a्a }}{ } \mathrm{M} 1994$.

UNE 127100:1999 “Tejas de hormigón. Código de práctica para la concepción y el montaje de cubiertas con tejas de hormigón".

UNE-EN 490:1995 "Tejas y accesorios de hormigón. Especificaciones de producto".

UNE-EN 491:1995 "Tejas y accesorios de hormigón. Métodos de ensayo".

UNE-EN 490:2005 "Tejas y piezas de hormigón para tejados y revestimiento de muros. Especificaciones de producto».

UNE-EN 491:2005 «Tejas y piezas de hormigón para tejados y revestimiento de muros. Métodos de ensayo».

UNE-EN-ISO 9001:2000 "Sistemas de gestión de la calidad. Requisitos".

Proyecto EN 13693 "Special precast prestressed concrete roof elements".

\section{AGRADECIMIENTOS}

El desarrollo de la normativa se ha realizado con el apoyo financiero del Proyecto de Investigación subvencionado FIT 030000-2001-131 I+D en materiales para la mejora de la composición de la teja de hormigón, investigación prenormativa, del Ministerio de Ciencia y Tecnología y las subvenciones a la normalización internacional de AENOR. 